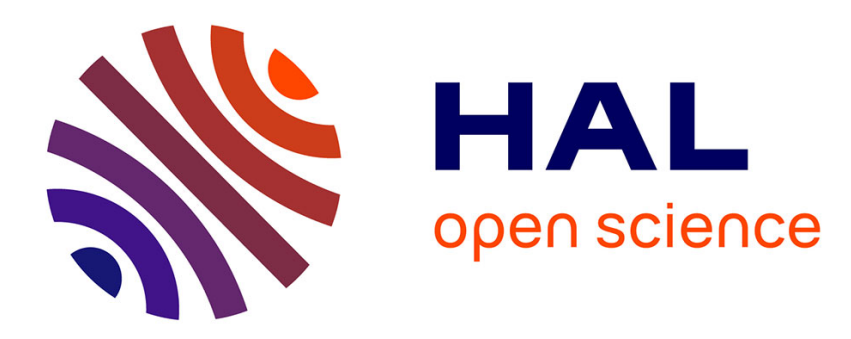

\title{
On the Kostant conjecture for Clifford algebra
}

\author{
Anton Alekseev, Anne Moreau
}

\section{To cite this version:}

Anton Alekseev, Anne Moreau. On the Kostant conjecture for Clifford algebra. 2011. hal-00639239

\section{HAL Id: hal-00639239 \\ https://hal.science/hal-00639239}

Preprint submitted on 8 Nov 2011

HAL is a multi-disciplinary open access archive for the deposit and dissemination of scientific research documents, whether they are published or not. The documents may come from teaching and research institutions in France or abroad, or from public or private research centers.
L'archive ouverte pluridisciplinaire HAL, est destinée au dépôt et à la diffusion de documents scientifiques de niveau recherche, publiés ou non, émanant des établissements d'enseignement et de recherche français ou étrangers, des laboratoires publics ou privés. 


\title{
ON THE KOSTANT CONJECTURE FOR CLIFFORD ALGEBRAS
}

\author{
ANTON ALEKSEEV AND ANNE MOREAU
}

\begin{abstract}
Let $\mathfrak{g}$ be a complex simple Lie algebra, and $\mathfrak{h} \subset \mathfrak{g}$ be a Cartan subalgebra. In the end of 1990s, B. Kostant defined two filtrations on $\mathfrak{h}$, one using the Clifford algebras and the odd analogue of the Harish-Chandra projection hc odd $: \mathrm{Cl}(\mathfrak{g}) \rightarrow \mathrm{Cl}(\mathfrak{h})$, and the other one using the canonical isomorphism $\check{\mathfrak{h}}=\mathfrak{h}^{*}$ (here $\check{\mathfrak{h}}$ is the Cartan subalgebra in the simple Lie algebra $\mathfrak{g}$ corresponding to the dual root system) and the adjoint action of the principal $\mathfrak{s l}_{2}$-triple $\check{\mathfrak{s}} \subset \check{\mathfrak{g}}$. Kostant conjectured that the two filtrations coincide.

The two filtrations arise in very different contexts, and comparing them proved to be a difficult task. Y. Bazlov settled the conjecture for $\mathfrak{g}$ of type $A_{n}$ using explicit expressions for primitive invariants in the exterior algebra $\wedge \mathfrak{g}$. Up to now this approach did not lead to a proof for all simple Lie algebras.

Recently, A. Joseph proved that the second Kostant filtration coincides with the filtration on $\mathfrak{h}$ induced by the generalized Harish-Chandra projection $(U \mathfrak{g} \otimes \mathfrak{g})^{\mathfrak{g}} \rightarrow S \mathfrak{h} \otimes \mathfrak{h}$ and the evaluation at $\rho \in \mathfrak{h}^{*}$. In this note, we prove that Joseph's result is equivalent to the Kostant Conjecture. We also show that the standard Harish-Chandra projection $U \mathfrak{g} \rightarrow S \mathfrak{h}$ composed with evaluation at $\rho$ induces the same filtration on $\mathfrak{h}$.
\end{abstract}

\section{INTRODUCTION}

Our starting point in this work is the fundamental paper by Kostant [K97] on the structure of Clifford algebras over complex simple Lie algebras. Let $G$ be a complex simple Lie group of rank $r$ and $\mathfrak{g}$ be the corresponding Lie algebra. The cohomology ring of $G$ (over $\mathbb{C}$ ) is isomorphic to the ring of bi-invariant differential forms, $H(G) \cong\left(\wedge \mathfrak{g}^{*}\right)^{\mathfrak{g}}$. By the Hopf-Koszul-Samelson theorem, $\left(\wedge \mathfrak{g}^{*}\right)^{\mathfrak{g}}$ is isomorphic to the exterior algebra $\wedge P$, where $P$ is a graded vector space with generators in degrees $2 m_{i}+1$ defined by the exponents of $\mathfrak{g}$, $m_{1}, \ldots, m_{r}$. For all $\mathfrak{g}$ simple, $m_{1}=1$ and the corresponding bi-invariant differential form is the Cartan 3-form

$$
\eta(x, y, z)=B_{\mathfrak{g}}(x,[y, z]),
$$

where $x, y, z \in \mathfrak{g}$ and $B_{\mathfrak{g}}$ is the unique (up to multiple) invariant scalar product on $\mathfrak{g}$.

The scalar product $B_{\mathfrak{g}}$ defines a structure of a Clifford algebra $\mathrm{Cl}(\mathfrak{g})=T \mathfrak{g} /\langle x \otimes x-$ $\left.B_{\mathfrak{g}}(x, x)\right\rangle$, where $T \mathfrak{g}$ is the tensor algebra of $\mathfrak{g}$. One of the main results of [K97] is the theorem stating that the adjoint invariant part of the Clifford algebra $\mathrm{Cl}(\mathfrak{g})^{\mathfrak{g}} \cong \mathrm{Cl}(P)$ is isomorphic to the Clifford algebra over $P$ (with the scalar product $B_{P}$ induced by $B_{\mathfrak{g}}$ ). Under this isomorphism, the Cartan 3-form defines a canonical cubic element $\hat{\eta} \in \mathrm{Cl}(\mathfrak{g})$. This element plays an important role in the theory of Kostant's cubic Dirac operator (see [K03] and [AM00]), in the theory of group valued moment maps [AMW00], and recently in the Chern-Simons theory in dimension one [AM11].

Let $\mathfrak{g}=\mathfrak{n}_{+} \oplus \mathfrak{h} \oplus \mathfrak{n}_{-}$be a triangular decomposition of $\mathfrak{g}$, and let $\theta: \mathfrak{g} \rightarrow \mathrm{Cl}(\mathfrak{g})$ be the canonical injection of $\mathfrak{g}$ as the generating set of $\mathrm{Cl}(\mathfrak{g})$. Consider the direct sum decomposition of the Clifford algebra

$$
\mathrm{Cl}(\mathfrak{g})=\mathrm{Cl}(\mathfrak{h}) \oplus\left(\theta\left(\mathfrak{n}_{-}\right) \mathrm{Cl}(\mathfrak{g})+\operatorname{Cl}(\mathfrak{g}) \theta\left(\mathfrak{n}_{+}\right)\right)
$$


and the odd Harish-Chandra projection $\mathrm{hc}_{\text {odd }}: \mathrm{Cl}(\mathfrak{g}) \rightarrow \mathrm{Cl}(\mathfrak{h})$. The map hc odd is important in various applications (e.g. in the localization formulas of [AMW00]). In particular, the image of the canonical cubic element $\hat{\eta}$ is given by formula,

$$
\operatorname{hc}_{\text {odd }}(\hat{\eta})=B_{\mathfrak{g}}^{\sharp}(\rho),
$$

where $\rho \in \mathfrak{h}^{*}$ is the half-sum of positive roots, and $B_{\mathfrak{g}}^{\sharp}: \mathfrak{h}^{*} \rightarrow \mathfrak{h}$ is the isomorphism induced by the scalar product $B_{\mathfrak{g}}$. In this context, the natural question is to evaluate the images of higher degree generators of $P$ under the map hc $\mathrm{c}_{\text {odd }}$. It is convenient to state this question in terms of the natural filtration of $P$ by degree (in contrast to grading, the filtration survives the passage to the Clifford algebra). A non-trivial result of Bazlov [B2] and Kostant (private communications) is $\operatorname{hc}_{\text {odd }}(P)=\theta(\mathfrak{h}) \subset \mathrm{Cl}(\mathfrak{h})$. Hence, $\operatorname{hc}_{\text {odd }}\left(P^{(k)}\right)$ defines a filtration on the Cartan subalgebra $\mathfrak{h}$.

Let $\check{\mathfrak{g}}$ be the Lie algebra defined by the dual root system, and $\check{\mathfrak{h}} \subset \check{\mathfrak{g}}$ the corresponding Cartan subalgebra. Note that there is a canonical isomorphism $\mathfrak{h}^{*} \cong \mathfrak{h}$. The key observation of Kostant is that $\rho \in \mathfrak{h}^{*}$ viewed as an element of $\check{\mathfrak{h}}$ coincides with the Cartan element $\check{h}$ of the principal sl $\mathrm{s}_{2}$-triple $(\check{e}, \check{h}, \check{f}) \subset \check{\mathfrak{g}}$. The action of this $\mathfrak{s l}_{2}$-triple induces a natural filtration of $\check{\mathfrak{h}}$,

$$
\mathcal{F}^{(m)} \check{\mathfrak{h}}=\left\{x \in \check{\mathfrak{h}}, \operatorname{ad}_{\check{e}}^{m+1} x=0\right\} .
$$

Since $[\check{e},[\check{e}, \check{h}]]=0$, we have $\rho=\check{h} \in \mathcal{F}^{(1)} \check{\mathfrak{h}}$. Kostant suggested the following conjecture:

Kostant Conjecture. The following two filtrations on $\mathfrak{h}$ coincide:

$$
\operatorname{hc}_{\text {odd }}\left(P^{(2 m+1)}\right)=\theta\left(B_{\mathfrak{g}}^{\sharp}\left(\mathcal{F}^{(m)} \check{\mathfrak{h}}\right)\right) .
$$

The two filtrations of the Kostant Conjecture arise in very different contexts, and comparing them proved to be a difficult task. In [B], Bazlov settled the Kostant conjecture for $\mathfrak{g}$ of type $A_{n}$ using explicit expressions for higher generators of $P$. The text [B2] claims to prove the conjecture for all simple Lie algebras $\mathfrak{g}$, but there is a gap in the argument (to be more precise, in Lemma 4.4).

The link between the two filtrations in the Kostant Conjecture can be established in two steps. The decisive step has been recently made by Joseph in $[\mathrm{J}]$. He proved that filtration $B_{\mathfrak{g}}^{\sharp}\left(\mathcal{F}^{(m)} \check{\mathfrak{h}}\right)$ arises in the context of generalized Harish-Chandra projections recently introduced in [KNV11]. In more detail, the generalized Harish-Chandra projection $\mathrm{hc}_{\mathfrak{g}}:(U \mathfrak{g} \otimes \mathfrak{g})^{\mathfrak{g}} \rightarrow S \mathfrak{h} \otimes \mathfrak{h}$ composed with $\mathrm{ev}_{\rho}: S \mathfrak{h} \rightarrow \mathbb{C}$, the evaluation at $\rho$, has the following property:

\section{Joseph's Theorem.}

$$
\left(\operatorname{ev}_{\rho} \otimes 1\right) \circ h c_{\mathfrak{g}}\left(U^{(m)} \mathfrak{g} \otimes \mathfrak{g}\right)^{\mathfrak{g}}=B_{\mathfrak{g}}^{\sharp}\left(\mathcal{F}^{(m)} \check{\mathfrak{h}}\right) .
$$

Here $U^{(m)} \mathfrak{g}$ is the natural filtration of the universal enveloping algebra by degree (where all elements of $\mathfrak{g} \subset U \mathfrak{g}$ are assigned degree one).

Our first result in this note is the equivalence of the Kostant Conjecture and Joseph's theorem. In more detail, we prove that

$$
\theta\left(\left(\operatorname{ev}_{\rho} \otimes 1\right) \circ \mathrm{hc}_{\mathfrak{g}}\left(U^{(m)} \mathfrak{g} \otimes \mathfrak{g}\right)^{\mathfrak{g}}\right)=\mathrm{hc}_{\text {odd }}\left(P^{(2 m+1)}\right) .
$$

This results settles the Kostant Conjecture for all $\mathfrak{g}$ simple. Our proof is a combination of elementary Clifford calculus and of deep results of [K97]. 
Our second result is a variation of the same theme. For the standard Harish-Chandra projection hc $: U \mathfrak{g} \rightarrow S \mathfrak{h}$, we prove that

$$
\theta\left(\left(\operatorname{ev}_{\rho} \circ \mathrm{hc} \otimes 1\right)\left(U^{(m)} \mathfrak{g} \otimes \mathfrak{g}\right)^{\mathfrak{g}}\right)=\mathrm{hc}_{\text {odd }}\left(P^{(2 m+1)}\right) .
$$

While this paper was in preparation, A. Joseph informed us that he proved the following theorem [J2]:

$$
\left(\mathrm{ev}_{\rho} \circ \mathrm{hc} \otimes 1\right)\left(U^{(m)} \mathfrak{g} \otimes \mathfrak{g}\right)^{\mathfrak{g}}=B_{\mathfrak{g}}^{\sharp}\left(\mathcal{F}^{(m)} \check{\mathfrak{h}}\right)
$$

Together with our result, it gives an alternative proof of the Kostant Conjecture.

Acknowledgments. We are indebted to B. Kostant for introducing us to the subject, and to E. Meinrenken for useful discussions and encouragement. We would like to thank A. Joseph for sharing with us the results of his paper [J2]. Our work was supported in part by the grants 200020-126817 and 200020-126909 of the Swiss National Science Foundation.

\section{PRincipal Filtrations ON $\mathfrak{h}$}

Let $\mathfrak{g}$ be a complex finite dimensional simple Lie algebra of rank $r$ and

$$
\mathfrak{g}=\mathfrak{n}_{-} \oplus \mathfrak{h} \oplus \mathfrak{n}_{+}
$$

a triangular decomposition of $\mathfrak{g}$. Let $\Delta \subset \mathfrak{h}^{*}$ be the root system of $(\mathfrak{g}, \mathfrak{h}), \Pi$ the system of simple roots with respect to the Borel subalgebra $\mathfrak{h} \oplus \mathfrak{n}_{+}$and $\Delta^{+}$the corresponding set of positive roots. For any $\alpha \in \Delta$, we denote by $\check{\alpha} \in \mathfrak{h}$ its coroot. Choose for each $\alpha \in \Delta$ a nonzero element $e_{\alpha}$ in the $\alpha$-root space so that $\left\{\check{\beta}, e_{\alpha} ; \beta \in \Pi, \alpha \in \Delta\right\}$ is a Chevalley basis of $\mathfrak{g}$.

Let $e=\sum_{\beta \in \Pi} e_{\beta} \in \mathfrak{n}_{+}$be the principal nilpotent element. By Jocobson-Morosov theorem, one can form an $\mathfrak{s l}_{2}$-triple $(e, h, f)$ with $h \in \mathfrak{h}$ and $f \in \mathfrak{n}_{-}$. The triple $(e, h, f)$ spans the principal $\mathfrak{s l}_{2}$ Lie subalgebra $\mathfrak{s} \subset \mathfrak{g}$. Using the adjoint action of $\mathfrak{s}$ on $\mathfrak{g}$, one can define a filtration of $\mathfrak{h}$,

$$
\mathcal{F}^{(m)} \mathfrak{h}=\left\{x \in \mathfrak{h}, \operatorname{ad}_{e}^{m+1} x=0\right\} .
$$

The dimension of the vector space $\mathcal{F}^{(m)} \mathfrak{h}$ jumps at the values $m=m_{1}, \ldots, m_{r}$, the exponents of $\mathfrak{g}$. This follows from the Kostant's theorem [K59] which shows that $\mathfrak{g}$ is a direct sum of $r$ irreducible $\operatorname{ad}_{\mathfrak{s}}$-modules of dimensions $2 m_{i}+1$. In most cases, the exponents $m_{1}, \ldots, m_{r}$ are all distinct. The exception is the case of the $D_{n}$ series, for even $n$ and $n \geqslant 4$, when there are two coincident exponents (equal to $n-1$ ).

The filtration $\mathcal{F}^{(m)} \mathfrak{h}$ is induced by a grading. In more detail, let $B_{\mathfrak{g}}$ be a unique (up to multiple) invariant scalar product on $\mathfrak{g}$. It restricts to a scalar product on $\mathfrak{h}$. The orthogonal complements $\mathcal{F}^{(m)} \mathfrak{h}^{\perp}$ form a new filtration. Define $\mathcal{F}_{m} \mathfrak{h}=\mathcal{F}^{(m)} \mathfrak{h} \cap \mathcal{F}^{(m-1)} \mathfrak{h}^{\perp}$. Note that $\mathcal{F}_{m} \mathfrak{h}$ are non-empty only for $m=m_{i}$ (and in most cases these are complex lines). Then, $\mathcal{F}^{(m)} \mathfrak{h}=\oplus_{k \leqslant m} \mathcal{F}_{k} \mathfrak{h}$.

Let $\check{\mathfrak{g}}$ be the simple Lie algebra corresponding to the dual root system $\check{\Delta}$ of $\Delta$. The Cartan subalgebra $\breve{h}$ (with respect to $\check{\Delta}$ ) of $\check{\mathfrak{g}}$ is canonically isomorphic to $\mathfrak{h}^{*}$. As a consequence, we can identify $\check{\mathfrak{h}}$ to $\mathfrak{h}^{*}$. Let $\check{e}=\sum_{\beta \in \Pi} \check{e}_{\breve{\beta}}$ be the principal nilpotent element of $\check{\mathfrak{g}}$ (here $\left\{\beta, \check{e}_{\check{\alpha}} ; \beta \in \Pi, \alpha \in \Delta\right\}$ is a Chevalley basis of $\check{\mathfrak{g}}$ ). Since $\check{\mathfrak{h}}^{*}$ and $\mathfrak{h}$ are canonically isomorphic, the co-adjoint action of $\check{e}$ induces another filtration on $\mathfrak{h}$,

$$
\check{\mathcal{F}}^{(m)} \mathfrak{h}=\left\{x \in \mathfrak{h},\left(\operatorname{ad}_{\check{e}}^{*}\right)^{m+1} x=0\right\} .
$$

The restriction of $B_{\mathfrak{g}}$ to $\check{\mathfrak{h}}$ is nondegenerate, hence it induces an isomorphism, $B_{\mathfrak{g}}^{b}$, between $\check{\mathfrak{h}}$ and $\check{\mathfrak{h}}^{*}$ and a nondegenerate bilinear form on $\check{\mathfrak{h}}^{*} \cong \mathfrak{h}$. Observe that $\check{\mathcal{F}}^{(m)} \mathfrak{h}=B_{\mathfrak{\mathfrak { g }}}^{\mathfrak{b}}\left(\check{\mathcal{F}}^{(m)} \check{\mathfrak{h}}\right)$ 
where $\check{\mathcal{F}}^{(m)} \check{\mathfrak{h}}=\left\{x \in \check{\mathfrak{h}}, \operatorname{ad}_{\check{e}}^{m+1} x=0\right\}$. Similarly to $\mathcal{F}^{(m)} \mathfrak{h}$, the filtration $\check{\mathcal{F}}^{(m)} \mathfrak{h}$ is induced by a grading, $\check{\mathcal{F}}^{(m)} \mathfrak{h}=\oplus_{k \leqslant m} \check{\mathcal{F}}_{k} \mathfrak{h}$, with $\check{\mathcal{F}}_{m} \mathfrak{h}=\check{\mathcal{F}}^{(m)} \mathfrak{h} \cap \check{\mathcal{F}}^{(m-1)} \mathfrak{h}^{\perp}$.

Remark 2.1. The bilinear forms $\left.B_{\mathfrak{g}}\right|_{\mathfrak{h} \times \mathfrak{h}}$ and $\left.B_{\mathfrak{g}}\right|_{\mathfrak{h} \times \mathfrak{h}}$ do not coincide in general but they are proportional to each other. Hence, the orthogonal complements are defined relative to $\left.B_{\mathfrak{g}}\right|_{\mathfrak{h} \times \mathfrak{h}}$ or, equivalently, to $\left.B_{\mathfrak{g}}\right|_{\mathfrak{h} \times \mathfrak{h}}$.

\section{Clifford algebra and odd Harish-Chandra projection}

Let $\mathrm{Cl}(\mathfrak{g})=\mathrm{Cl}\left(\mathfrak{g}, B_{\mathfrak{g}}\right)$ be the Clifford algebra defined by the scalar product $B_{\mathfrak{g}}$. Recall that $\mathrm{Cl}(\mathfrak{g})$ is the quotient of the tensor algebra $T \mathfrak{g}$ by the ideal generated by elements $x \otimes x-B_{\mathfrak{g}}(x, x), x \in \mathfrak{g}$. Denote by $x y$ the product in $\mathrm{Cl}(\mathfrak{g})$, for $x, y \in \mathrm{Cl}(\mathfrak{g})$, and by $\theta: \mathfrak{g} \rightarrow \mathrm{Cl}(\mathfrak{g})$ the canonical injection of $\mathfrak{g}$ as a generating subspace of $\mathrm{Cl}(\mathfrak{g})$.

As a vector space, $\mathrm{Cl}(\mathfrak{g})$ is isomorphic to the exterior algebra $\wedge \mathfrak{g}$, with an explicit $\operatorname{ad}_{\mathfrak{g}}$ equivariant isomorphism given by the anti-symmetrization map

$$
q: \wedge \mathfrak{g} \rightarrow \mathrm{Cl}(\mathfrak{g}) .
$$

Note that $q$ maps the natural filtration on $\wedge \mathfrak{g}$ (with all $x \in \mathfrak{g}$ of degree one) to the natural filtration on $\mathrm{Cl}(\mathfrak{g})$. Set $\mathrm{Cl}^{0}(\mathfrak{g})=q\left(\sum_{k} \wedge^{2 k} \mathfrak{g}\right)$ and $\mathrm{Cl}^{1}(\mathfrak{g})=q\left(\sum_{k} \wedge^{2 k+1} \mathfrak{g}\right)$. Then $\mathrm{Cl}^{i}(\mathfrak{g}) \mathrm{Cl}^{j}(\mathfrak{g}) \subset \mathrm{Cl}^{i+j}(\mathfrak{g})$ for $i, j \in\{0,1\}$, where we set $\mathrm{Cl}^{2}(\mathfrak{g})=\mathrm{Cl}^{0}(\mathfrak{g})$. Hence, $\mathrm{Cl}(\mathfrak{g})$ inherits the structure of a super associative algebra and so has the structure of a super Lie algebra, $[$,$] , defined by:$

$$
[u, v]=u v-(-1)^{i j} v u, \quad u \in \mathrm{Cl}^{i}(\mathfrak{g}), v \in \mathrm{Cl}^{j}(\mathfrak{g}) .
$$

Note that for $x, y \in \mathfrak{g}$, we have $[\theta(x), \theta(y)]=\theta(x) \theta(y)+\theta(x) \theta(y)=2 B_{\mathfrak{g}}(x, y)$. To avoid confusion, we will denote by $[,]_{\mathfrak{g}}$ the usual Lie bracket on $\mathfrak{g}$.

The scalar product $B_{\mathfrak{g}}$ extends to a scalar product on $\wedge \mathfrak{g}$, again denoted by $B_{\mathfrak{g}}$, in the standard way. Let $P \subset(\wedge \mathfrak{g})^{\mathfrak{g}}$ be the set of primitive element which is the $B_{\mathfrak{g}^{-}}$ orthocomplement to $(\wedge \mathfrak{g})_{+}^{\mathfrak{g}} \wedge(\wedge \mathfrak{g})_{+}^{\mathfrak{g}}$ in $(\wedge \mathfrak{g})_{+}^{\mathfrak{g}}$ where $(\wedge \mathfrak{g})_{+}^{\mathfrak{g}}$ is the augmentation ideal of $(\wedge \mathfrak{g})^{\mathfrak{g}}$. Let $\alpha$ be the unique anti-automorphism of $\wedge \mathfrak{g}$ which is equal to identity on $\mathfrak{g}$, and let $B_{P}$ be the bilinear form defined on $P \times P$ by $B_{P}(u, v)=B_{\mathfrak{g}}(\alpha u, v)$.

The set $P$ is a graded vector space of dimension $r$ with generators in degrees $2 m_{i}+1$, $i=1, \ldots, r$, see $[K 97, \S 4.3]$. We will be using the natural filtration

$$
P^{(k)}=\{v \in P, \operatorname{deg}(v) \leqslant k\}, \quad k \in \mathbb{N} .
$$

Again, one can introduce the grading with $P^{(k)}=\oplus_{l \leqslant k} P_{l}$ and $P_{k}=P^{(k)} \cap\left(P^{(k-1)}\right)^{\perp}$. The graded components $P_{k}$ are nonvanishing for $k=2 m_{i}+1, i=1, \ldots, r$. Set $\mathcal{P}=q(P)$ and define the filtration

Obviously, $q\left(P^{(k)}\right)=\mathcal{P}^{(k)}$.

$$
\mathcal{P}^{(k)}=\{v \in \mathcal{P}, \operatorname{deg}(v) \leqslant k\}, \quad k \in \mathbb{N} .
$$

One can easily show that there is the following decomposition

$$
\operatorname{Cl}(\mathfrak{g})=\operatorname{Cl}(\mathfrak{h}) \oplus\left(\theta\left(\mathfrak{n}_{-}\right) \operatorname{Cl}(\mathfrak{g})+\operatorname{Cl}(\mathfrak{g}) \theta\left(\mathfrak{n}_{+}\right)\right),
$$

where $\mathrm{Cl}(\mathfrak{h}) \subset \mathrm{Cl}(\mathfrak{g})$ is the subalgebra spanned by $\theta(\mathfrak{h})$. The projection hc odd $: \mathrm{Cl}(\mathfrak{g}) \rightarrow$ $\mathrm{Cl}(\mathfrak{h})$ with respect to this decomposition is called the odd Harish-Chandra projection. When restricted to $\operatorname{ad}_{\mathfrak{g}}$-invariant elements, the map hc $\mathrm{hdd}_{\text {odd }} \circ q$ is an isomorphism, see [B2, Theorem 4.1]. Bazlov ([B2, Proposition 4.5]) and Kostant (private communications) proved the following non-trivial property of the map hc $\mathrm{odd}_{\text {: }}$ :

Theorem 3.1 (Bazlov-Kostant). We have: $\mathrm{hc}_{\text {odd }} \circ q(P)=\theta(\mathfrak{h})$. 
The following conjecture is due to Kostant (see also [B2, §5.6]):

Conjecture 3.2 (Kostant). For any $k \in \mathbb{N}$, we have: $\operatorname{hc}_{\text {odd }}\left(\mathcal{P}^{(2 k+1)}\right)=\theta\left(\check{\mathcal{F}}^{(k)} \mathfrak{h}\right)$.

Remark 3.3. In fact, the original formulation of Kostant's conjecture is more precise. It states that hc $\mathrm{c}_{\text {odd }}\left(q\left(P_{2 k+1}\right)\right)=\theta\left(\check{\mathcal{F}}_{k} \mathfrak{h}\right)$. However, it is sufficient to prove the statement about filtrations as we explain below.

The map hc $\mathrm{codd}_{\mathrm{o}} \circ q: P \rightarrow \theta(\mathfrak{h})$ is an isomorphism of vector spaces, and the scalar product $B_{P}$ is mapped to $B_{\mathfrak{g}}$ in the following sense: Let $v_{1}, v_{2} \in P$ and $x_{1}, x_{2} \in \mathfrak{h}$ such that hc $\mathrm{c}_{\text {odd }}\left(q\left(v_{1}\right)\right)=\theta\left(x_{1}\right)$ and $\mathrm{hc}_{\text {odd }}\left(q\left(v_{2}\right)\right)=\theta\left(x_{2}\right)$ (see Theorem 3.1). Theorem B in [K97] states that $\mathrm{Cl}(\mathfrak{g})^{\mathfrak{g}} \cong \mathrm{Cl}\left(P, B_{P}\right)$ with isomorphism induced by the injection $q: P \rightarrow \mathrm{Cl}(\mathfrak{g})^{\mathfrak{g}}$. As a consequence, we have $\left[q\left(v_{1}\right), q\left(v_{1}\right)\right]=2 B_{P}\left(v_{1}, v_{2}\right)$. Since in addition the restriction of $\mathrm{hc}_{\text {odd }} \circ q$ to $\operatorname{ad}_{\mathfrak{g}}$-invariant elements is an isomorphism, we get

$$
\begin{aligned}
2 B\left(x_{1}, x_{2}\right) & =\left[\theta\left(x_{1}\right), \theta\left(x_{2}\right)\right]=\left[\operatorname{hc}_{\text {odd }}\left(q\left(v_{1}\right)\right), \operatorname{hc}_{\text {odd }}\left(q\left(v_{2}\right)\right)\right] \\
& =\operatorname{hc}_{\text {odd }}\left(\left[q\left(v_{1}\right), q\left(v_{2}\right)\right]\right)=2 B_{P}\left(v_{1}, v_{2}\right) .
\end{aligned}
$$

Hence, $\operatorname{hc}_{\text {odd }}\left(q\left(P^{(2 k+1)}\right)\right)=\theta\left(\check{\mathcal{F}}^{(k)} \mathfrak{h}\right)$ implies hc $\operatorname{codd}_{\text {ord }}\left(q\left(P^{(2 k+1)}\right)^{\perp}\right)=\theta\left(\check{\mathcal{F}}^{(k)} \mathfrak{h}^{\perp}\right)$. Note that $P^{(2 k)}=P^{(2 k-1)}$. Indeed, remember that the graded components $P_{k}$ are nonvanishing for $k=2 m_{i}+1, i=1, \ldots, r$. So,

$$
\begin{aligned}
\operatorname{hc}_{\text {odd }}\left(q\left(P_{2 k+1}\right)\right) & =\operatorname{hc}_{\text {odd }}\left(q\left(P^{(2 k+1)} \cap\left(P^{(2 k)}\right)^{\perp}\right)\right) \\
& =\operatorname{hc}_{\text {odd }}\left(q\left(P^{(2 k+1)} \cap\left(P^{(2 k-1)}\right)^{\perp}\right)\right)=\theta\left(\check{\mathcal{F}}^{(k)} \mathfrak{h} \cap \check{\mathcal{F}}^{(k-1)} \mathfrak{h}^{\perp}\right)=\theta\left(\check{\mathcal{F}}_{k} \mathfrak{h}\right) .
\end{aligned}
$$

\section{Joseph's TheOREM implies the Kostant CONJeCture}

Recall that $U \mathfrak{g}$ admits a decomposition,

$$
U \mathfrak{g}=S \mathfrak{h} \oplus\left(\mathfrak{n}_{-} U \mathfrak{g}+U \mathfrak{g} \mathfrak{n}_{+}\right) .
$$

It induces the Harish-Chandra projection hc $: U \mathfrak{g} \rightarrow S \mathfrak{h}$. By the Harish-Chandra theorem, the center of the universal enveloping algebra is isomorphic (under the map hc) to the ring $(S \mathfrak{h})^{\cdot W}$ where the Weyl group $W$ acts on $\mathfrak{h}^{*}$ by the shifted action,

$$
\lambda \mapsto w \cdot \lambda=(\lambda+\rho)^{w}-\rho, \quad w \in W,
$$

where $\lambda \mapsto \lambda^{w}$ stands for the usual $W$-action. Recently, generalized Harish-Chandra projections were introduced in [KNV11]. Let $V$ be a finite-dimensional $\mathfrak{g}$-module, and denote by $\sigma$ the algebra homomorphism $\sigma: U \mathfrak{g} \rightarrow \operatorname{End}(V)$. The space $U \mathfrak{g} \otimes V$ carries two commuting $\mathfrak{g}$-actions,

$$
\rho_{L}(x): a \otimes b \rightarrow x a \otimes b, \quad \rho_{R}(x): a \otimes b \rightarrow-a x \otimes b+a \otimes \sigma(x) b .
$$

Using these two actions, one defines a direct sum decomposition,

$$
U \mathfrak{g} \otimes V=S \mathfrak{h} \otimes V \oplus\left(\rho_{L}\left(\mathfrak{n}_{-}\right)(U \mathfrak{g} \otimes V)+\rho_{R}\left(\mathfrak{n}_{+}\right)(U \mathfrak{g} \otimes V)\right),
$$

see the equality (4) in [KNV11] which refers to [KO08, Proposition 3.3]. This decomposition defines the generalized Harish-Chandra projection,

$$
\mathrm{hc}_{V}: U \mathfrak{g} \otimes V \rightarrow S \mathfrak{h} \otimes V .
$$

For $V$ the trivial module, $\mathrm{hc}_{V}$ coincides with the standard Harish-Chandra projection hc.

Under the generalized Harish-Chandra projection, the invariant subspace $(U \mathfrak{g} \otimes V)^{\mathfrak{g}}$ for the diagonal action $\rho(x)=\rho_{L}(x)+\rho_{R}(x)$ injects into $S \mathfrak{h} \otimes V[0]$ (here $V[0] \subset V$ is the zero 
weight subspace relative to the action of $\mathfrak{h}$ ); see for instance [KNV11, §3]. In particular, for $V=\mathfrak{g}$ equipped with the adjoint action, one gets an injection

$$
\mathrm{hc}_{\mathfrak{g}}:(U \mathfrak{g} \otimes \mathfrak{g})^{\mathfrak{g}} \hookrightarrow S \mathfrak{h} \otimes \mathfrak{h} .
$$

For every element $\lambda \in \mathfrak{h}^{*}$, one can introduce an evaluation map, $\mathrm{ev}_{\lambda}: S \mathfrak{h} \rightarrow \mathbb{C}$, associating to a polynomial $p \in S \mathfrak{h} \cong \mathbb{C}\left[\mathfrak{h}^{*}\right]$ its value at $\lambda, \mathrm{ev}_{\lambda}(p)=p(\lambda)$. In particular, we will be interested in the evaluation at $\rho$, the half-sum of positive roots. Recently, Joseph $[\mathrm{J}]$ proved the following theorem:

Theorem 4.1 (Joseph). For any $m \in \mathbb{N}$, we have:

$$
\left(\operatorname{ev}_{\rho} \otimes 1\right) \circ \mathrm{hc}_{\mathfrak{g}}\left(\left(U^{(m)} \mathfrak{g} \otimes \mathfrak{g}\right)^{\mathfrak{g}}\right)=\check{\mathcal{F}}^{(2 m+1)} \mathfrak{h} .
$$

Here $U^{(m)} \mathfrak{g}$ is the natural filtration of $U \mathfrak{g}$ induced by assigning degree one to all elements of $\mathfrak{g} \subset U \mathfrak{g}$. The proof of Joseph's theorem involves the technique of Zhelobenko and Bernshtein-Gelfand-Gelfand operators.

Our first result in this note is the following theorem:

Theorem 4.2. Joseph's Theorem is equivalent to the Kostant conjecture.

The rest of this section is devoted to the proof of Theorem 4.2. To advance, we formulate several key propositions. Let

$$
\tau: U \mathfrak{g} \rightarrow \mathrm{Cl}(\mathfrak{g})
$$

be the unique algebra homomorphism defined by the properties $[\tau(x), \theta(y)]=\theta\left([x, y]_{\mathfrak{g}}\right)$ and $\operatorname{deg}(\tau(x))=2$, for $x, y \in \mathfrak{g}$. Let

$$
\mathrm{m}_{\mathrm{Cl}}: \mathrm{Cl}(\mathfrak{g}) \otimes \mathfrak{g} \rightarrow \mathrm{Cl}(\mathfrak{g}), a \otimes b \rightarrow a \theta(b)
$$

be the product map in the Clifford algebra, and

$$
\mu=\mathrm{m}_{\mathrm{Cl}} \circ(\tau \otimes 1): U \mathfrak{g} \otimes \mathfrak{g} \rightarrow \mathrm{Cl}(\mathfrak{g}) .
$$

Since $\tau$ has degree two, note that $\mu$ maps $U^{(m)} \mathfrak{g} \otimes \mathfrak{g}$ to $\mathrm{Cl}^{(2 m+1)}(\mathfrak{g})$. Our first proposition is the following:

Proposition 4.3. The subspaces $\mu\left(\rho_{L}\left(\mathfrak{n}_{-}\right)(U \mathfrak{g} \otimes \mathfrak{g})\right)$ and $\mu\left(\rho_{R}\left(\mathfrak{n}_{+}\right)(U \mathfrak{g} \otimes \mathfrak{g})\right)$ are contained in the kernel of the odd Harish-Chandra projection hc $\mathrm{cdd}_{\text {od }}$.

Proof. To start with, observe that $\tau\left(\mathfrak{n}_{-}\right) \subset \theta\left(\mathfrak{n}_{-}\right) \mathrm{Cl}(\mathfrak{g})$ and $\tau\left(\mathfrak{n}_{+}\right) \subset \mathrm{Cl}(\mathfrak{g}) \theta\left(\mathfrak{n}_{+}\right)$. Indeed, elements of $\tau\left(\mathfrak{n}_{-}\right)$(resp. $\tau\left(\mathfrak{n}_{+}\right)$) are of negative (resp. positive) weight under the adjoint $\mathfrak{h}$-action.

For the first subspace, we have

$$
\mu\left(\rho_{L}\left(\mathfrak{n}_{-}\right)(U \mathfrak{g} \otimes \mathfrak{g})\right) \subset \mathrm{m}_{\mathrm{Cl}}\left(\tau\left(\mathfrak{n}_{-}\right) \tau(U \mathfrak{g}) \otimes \mathfrak{g}\right) \subset \tau\left(\mathfrak{n}_{-}\right) \operatorname{Cl}(\mathfrak{g}) \subset \theta\left(\mathfrak{n}_{-}\right) \operatorname{Cl}(\mathfrak{g}) .
$$

For the second subspace, a more detailed analysis is needed: For $a \in U \mathfrak{g}$ and $x, b \in \mathfrak{g}$, one has,

$$
\begin{aligned}
\mu\left(\rho_{R}(x)(a \otimes b)\right) & =\mathrm{m}_{\mathrm{Cl}} \circ(\tau \otimes 1)\left(-a x \otimes b+a \otimes[x, b]_{\mathfrak{g}}\right) \\
& =-\tau(a) \tau(x) \theta(b)+\tau(a) \theta\left([x, b]_{\mathfrak{g}}\right)=-\tau(a) \theta(b) \tau(x) .
\end{aligned}
$$

Hence,

$$
\mu\left(\rho_{R}\left(\mathfrak{n}_{+}\right)(U \mathfrak{g} \otimes \mathfrak{g})\right) \subset \mathrm{Cl}(\mathfrak{g}) \tau\left(\mathfrak{n}_{+}\right) \subset \mathrm{Cl}(\mathfrak{g}) \theta\left(\mathfrak{n}_{+}\right) .
$$

By definition, both $\theta\left(\mathfrak{n}_{-}\right) \mathrm{Cl}(\mathfrak{g})$ and $\operatorname{Cl}(\mathfrak{g}) \theta\left(\mathfrak{n}_{+}\right)$are contained in the kernel of hc odd and the proposition follows. 
The next fact is proved for instance in Lemma 4.2 of [B2]. For convenience, we recall the proof.

Proposition 4.4. For any $p \in S \mathfrak{h}$, one has $\mathrm{hc}_{\mathrm{odd}}(\tau(p))=\mathrm{ev}_{\rho}(p)$. In particular, hc $\mathrm{c}_{\mathrm{odd}} \circ \tau$ maps $S \mathfrak{h} \subset U \mathfrak{g}$ to $\mathbb{C}$.

Proof. For $x \in \mathfrak{h}$, we have

$$
\tau(x)=\frac{1}{4} \sum_{\alpha \in \Delta_{+}} \theta\left(e_{\alpha}\right) \theta\left(\left[e_{-\alpha}, x\right]_{\mathfrak{g}}\right)+\theta\left(e_{-\alpha}\right) \theta\left(\left[e_{\alpha}, x\right]_{\mathfrak{g}}\right)=\frac{1}{4} \sum_{\alpha \in \Delta_{+}} \alpha(x)\left(2-2 e_{-\alpha} e_{\alpha}\right) .
$$

As a result,

$$
\operatorname{hc}_{\text {odd }}(\tau(x))=\frac{1}{2} \sum_{\alpha \in \Delta_{+}} \alpha(x)=\rho(x) .
$$

Since $\mathrm{hc}_{\text {odd }}$ is an algebra homomorpism on $\operatorname{ad}_{\mathfrak{h}}$-invariant elements ([B2, Lemma 2.4]), it follows that hc $\mathrm{codd}_{\mathrm{o}}(\tau(p))=\mathrm{ev}_{\rho}(p)$ for any $p \in S \mathfrak{h}$, as required.

Define two maps $\mu_{i}:(U \mathfrak{g} \otimes \mathfrak{g})^{\mathfrak{g}} \rightarrow \mathrm{Cl}(\mathfrak{h})$, for $i=1,2$, as follows. The first map,

$$
\mu_{1}:(U \mathfrak{g} \otimes \mathfrak{g})^{\mathfrak{g}} \rightarrow S \mathfrak{h} \otimes \mathfrak{h} \rightarrow \mathfrak{h} \rightarrow \mathrm{Cl}(\mathfrak{h}),
$$

is the composition $\theta \circ\left(\mathrm{ev}_{\rho} \otimes 1\right) \circ \mathrm{hc}$. The second map,

$$
\mu_{2}:(U \mathfrak{g} \otimes \mathfrak{g})^{\mathfrak{g}} \rightarrow \mathrm{Cl}(\mathfrak{g})^{\mathfrak{g}} \rightarrow \mathrm{Cl}(\mathfrak{h}),
$$

is the composition of $\mu: U \mathfrak{g} \otimes \mathfrak{g} \rightarrow \mathrm{Cl}(\mathfrak{g})$ and of the odd Harish-Chandra projection hc odd $: \mathrm{Cl}(\mathfrak{g})^{\mathfrak{g}} \rightarrow \mathrm{Cl}(\mathfrak{h})$.

Proposition 4.5. The maps $\mu_{1}$ and $\mu_{2}$ are equal to each other.

Proof. By Proposition 4.3, $\mu_{2}(\alpha)=\mu_{2}\left(\mathrm{hc}_{\mathfrak{g}}(\alpha)\right)$ for $\alpha \in(U \mathfrak{g} \otimes \mathfrak{g})^{\mathfrak{g}}$ (here we view $S \mathfrak{h} \otimes \mathfrak{h}$ as a subspace of $U \mathfrak{g} \otimes \mathfrak{g})$. Furthermore, writing hc $\mathfrak{g}_{\mathfrak{g}}(\alpha)=\sum_{k} a_{k} \otimes x_{k}$ with $a_{k} \in S \mathfrak{h}, x_{k} \in \mathfrak{h}$, one has

$$
\mu_{2}(\alpha)=\sum_{k} \mu_{2}\left(a_{k} \otimes x_{k}\right)=\sum_{k} \mathrm{hc}_{\mathrm{odd}} \circ \mathrm{m}_{\mathrm{Cl}} \circ(\tau \otimes 1)\left(a_{k} \otimes x_{k}\right)=\sum_{k} \mathrm{hc}_{\mathrm{odd}}\left(\tau\left(a_{k}\right) \theta\left(x_{k}\right)\right) .
$$

Recall that $\mathrm{hc}_{\text {odd }}$ is an algebra homomorphism on $\operatorname{ad}_{\mathfrak{h}}$-invariant elements. Hence, by Proposition 4.4, we get:

$$
\mu_{2}(\alpha)=\sum_{k} \mathrm{hc}_{\mathrm{odd}}\left(\tau\left(a_{k}\right)\right) \mathrm{hc}_{\mathrm{odd}}\left(\theta\left(x_{k}\right)\right)=\sum_{k} \operatorname{ev}_{\rho}\left(a_{k}\right) \theta\left(x_{k}\right)=\left(\operatorname{ev}_{\rho} \otimes \theta\right)\left(\mathrm{hc}_{\mathfrak{g}}(\alpha)\right)=\mu_{1}(\alpha) .
$$

Our last proposition is essentially due to Kostant [K97].

Proposition 4.6. For any $m \in \mathbb{N}$, we have $\mu\left(\left(U^{(m)} \mathfrak{g} \otimes \mathfrak{g}\right)^{\mathfrak{g}}\right)=\mathcal{P}^{(2 m+1)}$.

Proof. By Theorem D of [K97], $\mathrm{Cl}(\mathfrak{g})$ is a free module over the subalgebra $\mathrm{Cl}(\mathfrak{g})^{\mathfrak{g}}$. Namely,

$$
\mathrm{Cl}(\mathfrak{g})=\tau(U \mathfrak{g}) \otimes \mathrm{Cl}(\mathfrak{g})^{\mathfrak{g}} .
$$

Furthermore, $\tau$ maps the center $Z(U \mathfrak{g})$ of $U \mathfrak{g}$ to $\mathbb{C}$ (see also [K97, Corollary 36]). Corollary 80 and Theorem 89 of [K97] give an explicit expression for the elements in $\mathcal{P}^{\left(2 m_{i}+1\right)}$, $i=1, \ldots, r$ : For any $p \in \mathcal{P}^{\left(2 m_{i}+1\right)}$, we have

$$
p=\sum_{k} \tau\left(a_{k}\right) \theta\left(b_{k}\right),
$$


where $\sum_{k} a_{k} \otimes b_{k}$ is in $\left(U^{\left(m_{i}\right)} \mathfrak{g} \otimes \mathfrak{g}\right)^{\mathfrak{g}}$.

As a consequence, we obtain inclusions, $\mathcal{P} \subset \mu\left((U \mathfrak{g} \otimes \mathfrak{g})^{\mathfrak{g}}\right)$ and $\mathcal{P}^{\left(2 m_{i}+1\right)} \subset \mu\left(\left(U^{\left(m_{i}\right)} \mathfrak{g} \otimes\right.\right.$ $\left.\mathfrak{g})^{\mathfrak{g}}\right)$. The vector space $\mathcal{P}$ has dimension $r$ and $(U \mathfrak{g} \otimes \mathfrak{g})^{\mathfrak{g}}$ is a free module of rank $r$ over $Z(U \mathfrak{g})$. Then we deduce from the equality $\tau(Z(U \mathfrak{g}))=\mathbb{C}$ that $\mu\left((U \mathfrak{g} \otimes \mathfrak{g})^{\mathfrak{g}}\right)=\mathcal{P}$. Moreover, since $\operatorname{deg}(\tau(x))=2$ and $\operatorname{deg}(\theta(x))=1$ for $x \in \mathfrak{g}$, we get

$$
\mu\left(\left(U^{(m)} \mathfrak{g} \otimes \mathfrak{g}\right)^{\mathfrak{g}}\right) \subset \mathcal{P}^{(2 m+1)} .
$$

Hence, $\mu\left(\left(U^{(m)} \mathfrak{g} \otimes \mathfrak{g}\right)^{\mathfrak{g}}\right)=\mathcal{P}^{(2 m+1)}$.

We are now in the position to prove Theorem 4.2:

Proof of Theorem 4.2. Joseph's theorem (Theorem 4.1) states that

$$
\mu_{1}\left(\left(U^{(m)} \mathfrak{g} \otimes \mathfrak{g}\right)^{\mathfrak{g}}\right)=\theta\left(\check{\mathcal{F}}^{(m)} \mathfrak{h}\right) .
$$

We have to show that the above equality implies the Kostant conjecture (Conjecture 4.2). In other words, we have to show that $\mu_{1}\left(\left(U^{(m)} \mathfrak{g} \otimes \mathfrak{g}\right)^{\mathfrak{g}}\right)=h c_{\text {odd }}\left(\mathcal{P}^{(2 m+1)}\right)$. But by Proposition 4.5 and Proposition 4.6, we have

$$
\mu_{1}\left(\left(U^{(m)} \mathfrak{g} \otimes \mathfrak{g}\right)^{\mathfrak{g}}\right)=\mu_{2}\left(\left(U^{(m)} \mathfrak{g} \otimes \mathfrak{g}\right)^{\mathfrak{g}}\right)=\operatorname{hc}_{\text {odd }}\left(\mathcal{P}^{(2 m+1)}\right) .
$$

\section{More on Harish-Chandra PROJECTIONS}

The standard Harish-Chandra projection hc $: U \mathfrak{g} \rightarrow S \mathfrak{h}$ is equivariant under the adjoint action of $\mathfrak{h}$. Hence, the image of $(U \mathfrak{g} \otimes \mathfrak{g})^{\mathfrak{g}}$ under hc $\otimes 1$ lands in $S \mathfrak{h} \otimes \mathfrak{h}$. Our second result is the following equality of filtrations on $\mathfrak{h}$ :

\section{Theorem 5.1.}

$$
\left(\mathrm{ev}_{\rho} \circ \mathrm{hc} \otimes 1\right)\left(U^{(m)} \mathfrak{g} \otimes \mathfrak{g}\right)=\mathrm{hc}_{\mathrm{odd}}\left(\mathcal{P}^{(2 m+1)}\right) .
$$

We first state an auxiliary statement preparing the proof of this Theorem:

Proposition 5.2. The image of the map hc $-1: U \mathfrak{g} \rightarrow U \mathfrak{g}$ is contained in the kernel of $\mathrm{hc}_{\text {odd }} \circ \tau: U \mathfrak{g} \rightarrow \mathrm{Cl}(\mathfrak{h})$.

Proof. The image of the map hc -1 is the space $\mathfrak{n}_{-} U \mathfrak{g}+U \mathfrak{g} \mathfrak{n}_{+} \subset U \mathfrak{g}$. For the first term, we have

$$
\tau\left(\mathfrak{n}_{-} U \mathfrak{g}\right)=\tau\left(\mathfrak{n}_{-}\right) \tau(U \mathfrak{g}) \subset \theta\left(\mathfrak{n}_{-}\right) \mathrm{Cl}(\mathfrak{g}) \subset \operatorname{ker}\left(\mathrm{hc}_{\text {odd }}\right) .
$$

Indeed, as already observed, we have $\tau\left(\mathfrak{n}_{-}\right) \subset \theta\left(\mathfrak{n}_{-}\right) \mathrm{Cl}(\mathfrak{g})$ (see the proof of Proposition 4.3). Similarly, for the second term,

$$
\tau\left(U \mathfrak{g} \mathfrak{n}_{+}\right)=\tau(U \mathfrak{g}) \tau\left(\mathfrak{n}_{+}\right) \subset \operatorname{Cl}(\mathfrak{g}) \theta\left(\mathfrak{n}_{+}\right) \subset \operatorname{ker}\left(\mathrm{hc}_{\text {odd }}\right) .
$$

Now we are ready to present the proof of Theorem 5.1.

Proof of Theorem 5.1. By Proposition 5.2, the following maps from $U \mathfrak{g}$ to $\mathrm{Cl}(\mathfrak{h})$ coincide:

$$
\text { hc }_{\text {odd }} \circ \tau=\text { hc }_{\text {odd }} \circ \tau \circ \text { hc. }
$$

In turn, by Proposition 4.4, $\mathrm{hc}_{\text {odd }} \circ \tau=\mathrm{ev}_{\rho}$ on the image of the Harish-Chandra map hc $: U \mathfrak{g} \rightarrow S \mathfrak{h}$. Hence, we obtain an equality of maps:

$$
\text { hc }_{\text {odd }} \circ \tau=\operatorname{ev}_{\rho} \circ \text { hc. }
$$


This observation shows that the image of both maps is in fact equal to $\mathbb{C} \subset \mathrm{Cl}(\mathfrak{h})$.

For $x \in \mathfrak{g}$ define a linear form, $\beta_{x}: \mathfrak{g} \rightarrow \mathbb{C}$, given by $\beta_{x}(y)=B_{\mathfrak{g}}(x, y)$. Then, for all $x \in \mathfrak{h}$ and $\alpha \in(U \mathfrak{g} \otimes \mathfrak{g})^{\mathfrak{g}}$, we have

$$
\left(\mathrm{hc}_{\mathrm{odd}} \circ \tau \otimes \beta_{x}\right)(\alpha)=\left(\mathrm{ev}_{\rho} \circ \mathrm{hc} \otimes \beta_{x}\right)(\alpha) .
$$

For $x \in \mathfrak{h}, v \in \mathcal{P}$, consider the expression

$$
\left[\theta(x), \operatorname{hc}_{\text {odd }}(v)\right]=\operatorname{hc}_{\text {odd }}([\theta(x), v]) .
$$

By Theorem 3.1, $\operatorname{hc}_{\text {odd }}(v) \in \theta(\mathfrak{h})$ and the above expression is equal to $B_{\mathfrak{g}}(x, y)$, where $\operatorname{hc}_{\text {odd }}(v)=\theta(y)$. Assume that it vanishes for all $v \in \mathcal{P}^{(2 m+1)}$. By definition, it is equivalent to $x \in \mathrm{hc}_{\text {odd }}\left(\mathcal{P}^{(2 m+1)}\right)^{\perp}$.

By Theorem $\mathrm{F}$ (equality (n)) and Corollary 80 (equality (302)) in [K97], for any $v \in$ $\mathcal{P}^{(2 m+1)}$ there is an element $\alpha \in\left(U^{(m)} \mathfrak{g} \otimes \mathfrak{g}\right)^{\mathfrak{g}}$ such that $[\theta(x), v]=\left(\tau \otimes \beta_{x}\right)(\alpha)$. Furthermore, the space $\left(U^{(m)} \otimes \mathfrak{g}\right)^{\mathfrak{g}}$ surjects on $\left[\theta(x), \mathcal{P}^{(2 m+1)}\right]$ under the map $\tau \otimes \beta_{x}: U \mathfrak{g} \otimes \mathfrak{g} \rightarrow \operatorname{Cl}(\mathfrak{g})$ for $m \in \mathbb{N}$. Then,

$$
\mathrm{hc}_{\text {odd }}([\theta(x), v])=\left(\mathrm{hc}_{\mathrm{odd}} \circ \tau \otimes \beta_{x}\right)(\alpha)=\left(\mathrm{ev}_{\rho} \circ \mathrm{hc} \otimes \beta_{x}\right)(\alpha)=B\left(x,\left(\mathrm{ev}_{\rho} \circ \mathrm{hc} \otimes 1\right)(\alpha)\right) .
$$

Vanishing of this expression for any $\alpha \in\left(U^{(m)} \mathfrak{g} \otimes \mathfrak{g}\right)^{\mathfrak{g}}$ is equivalent to $x \in\left(\left(\mathrm{ev}_{\rho} \circ \mathrm{hc} \otimes\right.\right.$ 1) $\left.\left(U^{(m)} \mathfrak{g} \otimes \mathfrak{g}\right)^{\mathfrak{g}}\right)^{\perp}$.

We have shown that the orthogonal complements of the two filtrations coincide. Hence, so do the filtrations in question.

Remark 5.3. While this paper was in preparation, Joseph informed us that he proved [J2] (using Zhelobenko invariants) the following equality of filtrations:

$$
\left(\mathrm{ev}_{\rho} \circ \mathrm{hc} \otimes 1\right)\left(U^{(m)} \mathfrak{g} \otimes \mathfrak{g}\right)=\check{\mathcal{F}}^{(m)} \mathfrak{h} .
$$

Together with Theorem 5.1, this gives an alternative proof of the Kostant Conjecture.

Joseph's result resembles of an earlier result by Rohr [R10] who showed that the filtrations $\check{\mathcal{F}}^{(m)} \mathfrak{h}$ coincides with the filtration $\left(\operatorname{ev}_{\rho} \circ \Phi \otimes 1\right)\left(\left(S^{(m)} \mathfrak{g} \otimes \mathfrak{g}\right)^{\mathfrak{g}}\right)$, where $\Phi$ is the projection map onto $S \mathfrak{h}$ with respect to the decomposition,

$$
S \mathfrak{g}=S \mathfrak{h} \oplus S \mathfrak{g}\left(\mathfrak{n}_{-} \oplus \mathfrak{n}_{+}\right) .
$$

In fact, Rohr's result is an essential step in the proof of Joseph's theorem.

\section{REFERENCES}

[AM00] A. Alekseev and E. Meinrenken, The non-commutative Weil algebra, Invent. math. 139 (2000), no. $1,135-172$.

[AMW00] A. Alekseev, E. Meinrenken and C. Woodward, Group-valued equivariant localization, Invent. math. 140 (2000), no. 2, 327-350.

[AM11] A. Alekseev and P. Mnev, One-dimensional Chern-Simons theory, Commun. Math. Phys. 307 (2011), 185-227.

[B] Y. Bazlov, Exterior powers of the adjoint representation of a simple Lie algebra, $\mathrm{PhD}$ Thesis, Weizmann Institute, 2003.

[B2] Y. Bazlov, The Harish-Chandra isomorphism for Clifford algebras, preprint arXiv:0812.2059.

$[\mathrm{J}] \quad$ A. Joseph, Zhelobenko invariants, Bernstein-Gelfand-Gelfand operators and the analogue Kostant Clifford algebra conjecture, preprint arXiv:1109.5854.

[J2] A. Joseph, Analogue Zhelobenko invariants for the Kostant and Hitchin Clifford algebra conjectures, in preparation.

[KNV11] S. Khoroshkin, M. Nazarov and E. Vinberg, A generalized Harish-Chandra isomorphism, Adv. Math. 226 (2011), 1168-1180. 
[KO08] S.Khoroshkin and O.Ogievetsky, Mickelsson algebras and Zhelobenko operators, J. Algebra 319 (2008), 2113-2165.

[K59] B. Kostant, The principal three-dimensional subgroup and the Betti numbers of a compex simple Lie group, Amer. J. Math. 81 (1959), 973-1032.

[K97] B. Kostant, Clifford Algebra Analogue of the Hopf-Koszul-Samelson Theorem, the $\rho$-Decomposition on $C(\mathfrak{g})=$ End $V_{\rho} \otimes C(P)$, and the $\mathfrak{g}$-module Structure on $\wedge \mathfrak{g}$, Adv. Math. 125 (1997), 275-350.

[K03] B. Kostant, Dirac Cohomology for the Cubic Dirac Operator, Studies in memory of Issai Schur (Chevaleret/Rehovot, 2000), 69-93, Progr. Math., 210, Birkhäuser Boston, 2003.

[R10] R. Rohr, Principal basis in Cartan subalgebra, J. Lie Theory 20 (2010), no. 4, 673-687.

Section de mathématiques, Université de Genève, 2-4 Rue du Lièvre, C.P. 64, 1211 Genève 4, Switzerland

E-mail address: Anton.Alekseev@unige.ch

Laboratoire de Mathématiques et Applications, Université de Poitiers, Téléport 2, 11 Boulevard Marie et Pierre Curie, BP 30179, 86962 Futuroscope Chasseneuil Cedex, France

E-mail address: Anne.Moreau@math.univ-poitiers.fr 